\title{
Scanning Near-field Raman Spectroscopic Microscope
}

\author{
Sumio Hosaka \\ Gunma University \\ Japan
}

\section{Introduction}

Recently, a need to develop a high-speed semiconductor device increases remarkably. In order to achieve such a device characteristic, we have paid attentions to a device with localized stress such as Si-Ge device (Welser et al. 1994, Rim et al. 2000, Tezuka at al. 2001). Electron and hole mobility can be influenced by the stress distribution of active layer in very fine device. Thus, we have to control the strength of the stress as 2- or 3-dimensional distribution for development of advanced devices. It is very important to measure and analyze the stress of very fine semiconductor devices. We have expected to analyze the stress with Raman spectroscopy. In Raman spectrum, strong stokes and anti-stokes scattering peak shifts occur when compressing and stretching the local area of the device.

On the other hand, a minimum size of semiconductor device pattern will be miniaturized to reach to a region of $25 \mathrm{~nm}$ in the future. We have to analyze the stress distribution for such a structure with very fine probe in Raman spectroscopy. In optical microscopic Raman spectroscopy, there exists a diffraction limit so that we cannot obtain the spectrum with a resolution of less than submicron. In order to overcome the diffraction limit, scanning nearfield optical microscopy (SNOM) with a resolution of less than wavelength of optical probe by small aperture has been proposed by E. H. Synge in 1932. Then, SNOM technology with a few 10s nm resolution has reported by D. Pohl in 1986 (Durig et al., 1986, Fischer et al. 1989). The technology has been based on near-field optical probe with small aperture on the metal-coated probe or fiber probe (Trautman et al. 1994). On the other hand, another approach, using apertureless near-field optics, has been proposed by J. Wessel in 1985. The concept is based on surface enhanced Raman spectroscopy (SERS). Since 1985, SERS and SNOM technologies have been advanced by using scanning tunneling microscopy (STM) or atomic force microscopy (AFM) technique. So far, many researchers have reported lots of works using various type near field optical microscopes.

In Raman spectroscopy, the significant technical issuer is how to detect too weak Raman scattering signal to increase spatial resolution. For improving the signal, a tip-enhanced Raman spectroscopy (TERS) has been proposed by A. Hartschuh, L. Novotny et al. in 2003. Then, many researchers proposed various type TERSes; bottom illumination (Stockle et al., 2000, Hayazawa et al.,2003), side-illumination (Nieman et al., 2001, Hayazawa et al. 2002, Mehtani et al. 2005), and modified top illumination (Poborchii et al.. 2005). The concepts are based on SERS, in which enhancement of the signal is obtained in the vicinity of the metal 
particle or tip. It can use the metal particle or metal tip as Raman scattering near-field probe with larger amplitude of Raman scattering. Although TERS technology has high spatial resolution based on Raman scattering signal enhancement in the vicinity of metal particle, the metal particle or metal tip has serious problem that they are one of contamination sources in Si device fabrication process.

In SNOM or NSOM (near-field scanning optical microscopy), many researchers have also reported these technologies such as illumination mode, collection mode, illuminationcollection mode, etc (Pohl, 1986, Trautman et al. 1994). They are based on formation of fine electromagnetic field probe in the vicinity of small aperture on the metal tip as near-field optical probe. Although the aperture size controls the size of near-field optical probe, microscopic image is determined by a signal and noise ratio. Both probe size and optical power throughput are very important. So far, good spatial resolution was not demonstrated using aperture type SNOM (Hosaka et al., 1996, Ono et al., 2005).

In near-field Raman spectroscopy, M. Yoshikawa et al. have reported to develop near-field optical Raman spectroscopy with illumination-correction mode and fine aperture pyramidal probe (Yoshikawa et al., 2006). Using resonant Raman scattering, they have 2-dimensional stress distribution of the VLSI standard sample made by $\mathrm{Si}$ and $\mathrm{SiO}_{2}$ for checking AFM The resolution is, however, not so high as about $250 \mathrm{~nm}$ even though they employed near-field light in Raman spectroscopy, though the Raman peak shift image is improved rather than optical microscopic Raman spectroscopy. In S. Hosaka group SNOM research, thus resolution could be also a little improved with near-field light in a case of using metal aperture probe in illumination-correction mode SNOM. The group have already pointed out optical aperture probe has limited to improve a spatial resolution because the optical probe has two components of near-field and far-field optical probes (Hosaka et al., 1999). The farfield optical probe power is gigantic larger than that of near-field probe to eliminate the near-field optical probe. Recently, $10 \mathrm{~nm}$-less spatial resolution using surface plasmon effect near-field optical probe (Fischer et al., 1989) in an illumination-collection and depolarization mode SNOM (Hosaka et al., 2007).

The metal aperture on outside at the top of the pyramidal probe causes metal contamination on the device surface as described above. We have an idea to improve to get fine near-field optical probe and to protect the contamination in order to solve these problems. The idea is to utilize such a structure that the optical probe is made by plasmon resonance without the outside metal and its aperture. The probe is based on the commercial pyramidal probe. The outside and inside layers are made of insulator of $\mathrm{Si}_{3} \mathrm{~N}_{4}$ or $\mathrm{SiO}_{2}$ and metal, respectively. The structure can protect from the contamination. However, we are anxious if we get fine nearfield optical probe without the aperture. It is very interested to study the aperture-less pyramidal probe for high resolution image (Hosaka et al., 2007).

In this section, I describe recent research states of TERS and SNOM technologies. Then, I describe SNOM technology in regard to plasmon resonace optical probe using apertureless cantilever, depolarization optical system, Raman spectroscopy, etc. I describe estimation of near-field light propagation from the aperture-less pyramidal probe using FDTD method. And I describe some features of prototyped atomic force cantilevered SNOM with the aperture-less pyramidal probe and its combination system with Raman spectroscopy, and discuss on the possibility to detect Raman spectra for measuring fine stress distribution of semiconductor devices. 


\section{Raman scattering, TERS and SNOM technologies}

\subsection{Raman scattering}

When light incidents the sample, the reflected light is modulated by lattice vibration of the sample. Assuming that polarizability of the sample is given by $a=a_{0}+a_{1} \cos 2 \pi v$ s $t$ and the electric field is given by $E=E_{0} \cos 2 \pi v t$ the induced dipole moment $P$ is given by $P=a E$ where $v_{1}$ and $v$ are vibrations of the sample and incident light, respectively. The $\mathrm{P}$ is given by Eq. (1).

$$
\begin{aligned}
& P=a E=\left(a_{0}+a_{1} \cos 2 \pi v_{s} t\right) E_{o} 2 \pi v t \\
& =a_{0} E_{0} \cos 2 \pi v t+\frac{1}{2} a_{1} E_{0} \cos 2 \pi\left(v-v_{s}\right) t+\frac{1}{2} a_{1} E_{0} \cos 2 \pi\left(v+v_{s}\right) t
\end{aligned}
$$

The reflected dipole moment has 3 components as presented in above equation. The 1st, the 2nd and the 3rd terms correspond to Rayleigh scattering light, stokes scattering light and anti-stokes scattering light, respectively, as shown in Fig. 1(a). Therefore, stokes and antistokes scattering lights have the sample's information of lattice vibration, polarizability, atomic binding, stress, etc.

Furthermore, when compression or tensile stress is applied to the sample, the frequencies of stokes and anti-stokes scattering peaks are shifted. The peak-frequency shift (peak shift) occurs as shown in Fig. 1(b). The peak shifts to low and high frequencies mean tensile stress and compression stress, respectively. The stress $\sigma$ can be estimated from the shift $\Delta v$ as represented by Eq. (2).

$$
\sigma=2.3 \times 10^{4} \Delta v\left[\mathrm{Ncm}^{-2}\right]
$$

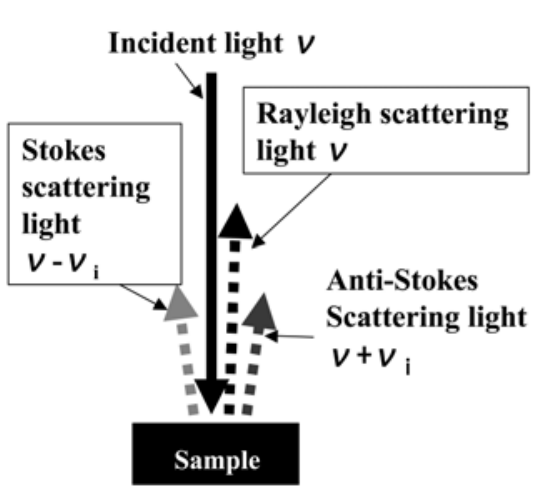

(a)

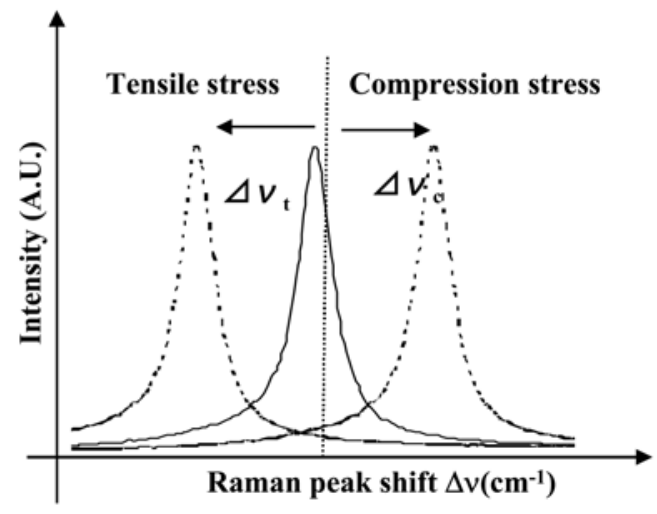

(b)

Fig. 1. Raman scattering phenomenon (a) and Raman peak shifts due to tensile andcompression stress (b).

\subsection{TERS}

The TERS has typically 3 types of bottom illumination, side illumination and modified top illumination as shown in Fig. 2. A. Hartschuh et al. have reported that near-field Raman spectroscopy with a spatial resolution of $20 \mathrm{~nm}$ has been demonstrated using a bottom 


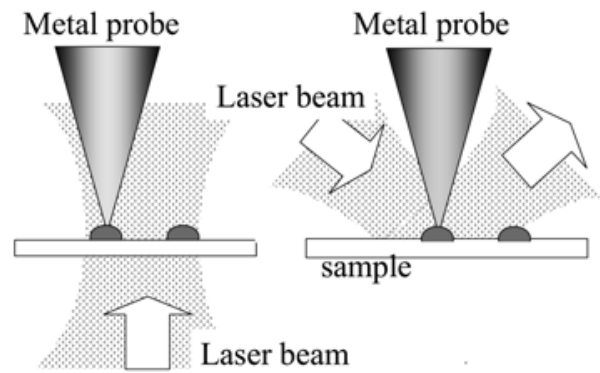

(a)

(b)

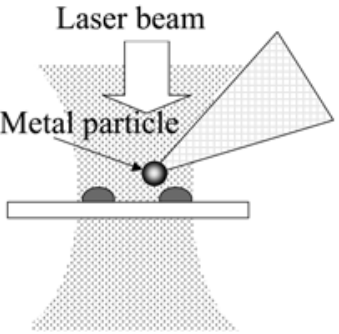

(c)

Fig. 2. Schematic diagrams of some TERSes; (a) bottom illumination, (b) side illuminationand (c) modified top illumination.

illumination mode TERS with vibration mode of single-wall carbon nanotubes (SWNTs) (Hartschuh et al., 2003). The tip material was gold. The tip was controlled within 10-50 pN using tuning fork detection in AFM. They demonstrated an enhancement of TERS signals of $G$ and $G^{\prime}$ bands by a comparison of near-field and far-field as shown in Fig. 3. They showed Raman scattering image of SWNT using $G^{\prime}$ band peak, and topographic and Raman scattering signal profiles (Fig. 4). The $G^{\prime}$ band Raman signal profile indicated that the spatial resolution was less than $30 \mathrm{~nm}$ because a FWHM of SWNT with $\mathrm{G}^{\prime}$ band peak was about 26 nm.

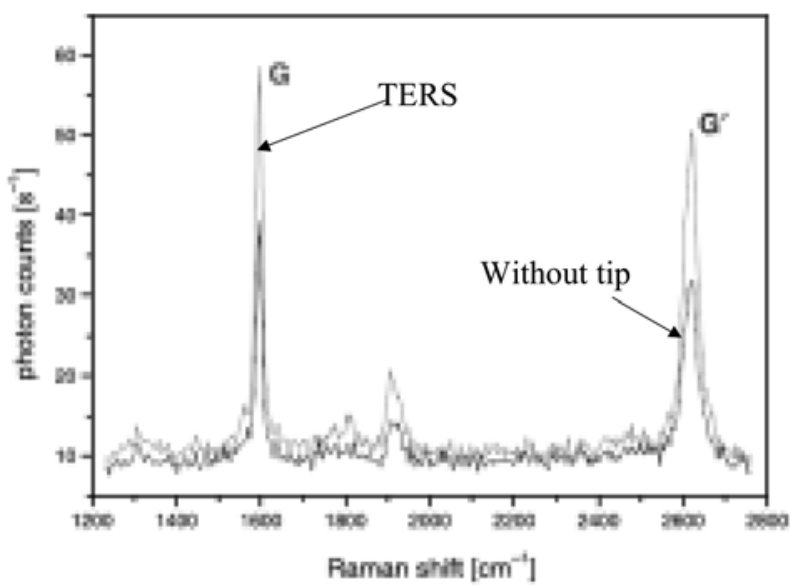

Fig. 3. Raman spectra detected with a sharp metal tip (green line) on top of the sample(distance; $1 \mathrm{~nm}$ ) and with the tip retracted by $2 \mu \mathrm{m}$ (black line). Note, the intensities of allRaman bands are increased with the tip close to the SWNTs (Hartschuh et al., 2003).

D. Mehtani et al. have introduced great potential of side illumination mode TERS for nanoscale chemical characterization and semiconductor (Mehtani et al., 2005). They demonstrated enhancement of Raman scattering signal of various molecular, polymeric and semiconducting materials as well as carbon nanotube (CNT) by comparing with far-field Raman signal. V. Poborchii et al. have introduced modified top illumination mode TERS using a silver particle on the top of quartz AFM cantilever probe immersed into glycerol 


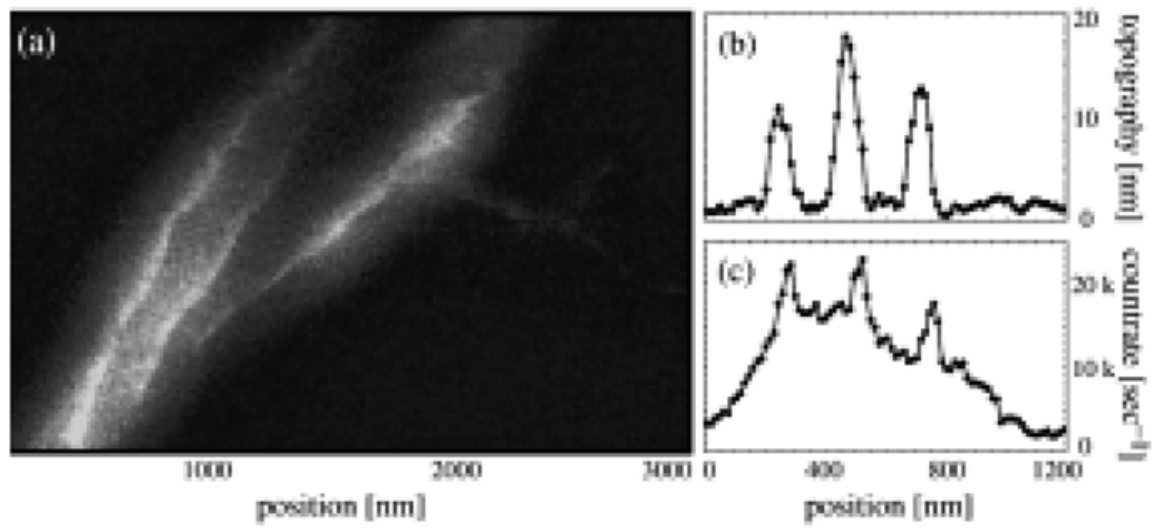

Fig. 4. (a) Raman image of SWNT bundles acquired by raster scanning a sharp metal tipand detecting the intensity of the $\mathrm{G}^{\prime}$ band (scan area $3 \times 1 \mu \mathrm{m}$, integration time 5 msper pixel). (b and c): Cross-sections taken along the white dotted line in (a) fortopography (b) and Raman signal (c) (Hartschuh et al., 2003).

droplet on Si surface (Poborchii et al.. 2005). As the experimental result, spatial resolution in a range of $100 \mathrm{~nm}$ was demonstrated. The system was used with depolarization optics without $364 \mathrm{~nm}$ primary light. As described above, TERS technology has tremendous potential to enhance Raman scattering signal compared with far-field Raman scattering signal, but the best spatial resolution was about $30 \mathrm{~nm}$. It is not enough to apply the technology to measure the Si device.

\subsection{SNOM}

A research on this technology has been focused into improvement of near-field optical probe with very fine probe and high contrast against far-field light. At first, S. Hosaka et al. changed the optical fiber probe to the AFM cantilevered pyramidal optical probe because the fiber probe was broken by hard contact between the probe and sample. The cantilevered optical probe has a possibility to observe nanometer-sized pits formed by electron beam (EB) drawing. They succeeded in observing the $30 \mathrm{~nm} \times 160 \mathrm{~nm}$ small pits by using the polarized near-field light as the illumination-collection mode SNOM. In the experiments, when they adopt an optical aperture on the cantilever, they had to focus illuminating laser beam into the aperture and to achieve another laser beam deflection optics for atomic force detection (optical lever). To achieve the requirements, they had to develop a through the lens (TTL) type optical lever. However, they have reported that illumination mode SNOM with an aperture on the top of metal probe has 2 components of near-field and far-field probes on the vicinity of the aperture (Fig. 5). The near-field probe power was very small rather than that of far-field probe. We needed to remove the far-field light and enhance the near-field probe power (high throughput) with small diameter. For the former, we adopted depolarization optics to detect only reflected near-field light without the far-field light reflected from the pyramidal probe. The illumination-collection mode SNOM optics was developed as shown in Fig. 6. The system obtained a spatial resolution of $300 \mathrm{~nm}$ from nearfield Kerr effect image of perpendicular magnetic recorded bits (Fig. 7). The resolution was not enough to obtain nanometer resolution by using such optics and probe. The low 

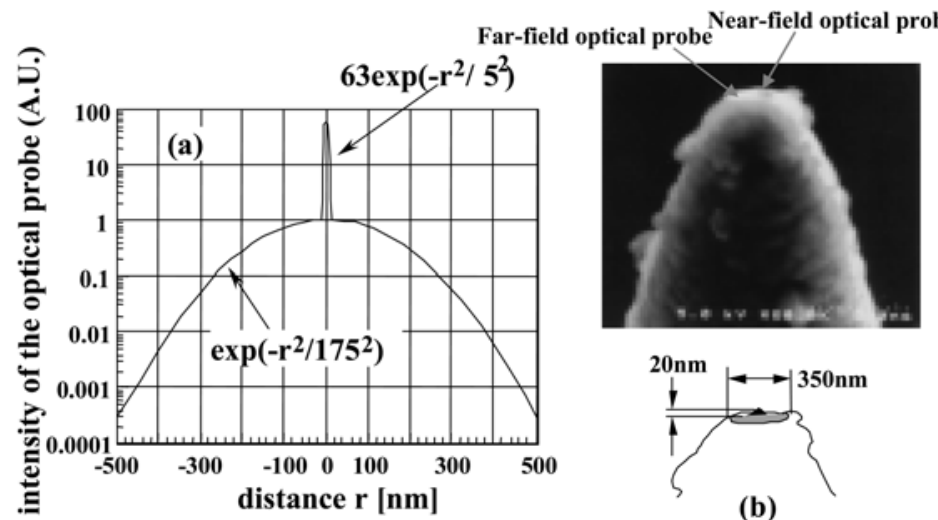

(b)

Fig. 5. Estimated optical probe profile (a) and the tip structure and its SEM image (b)(Hosaka et al., 1999).

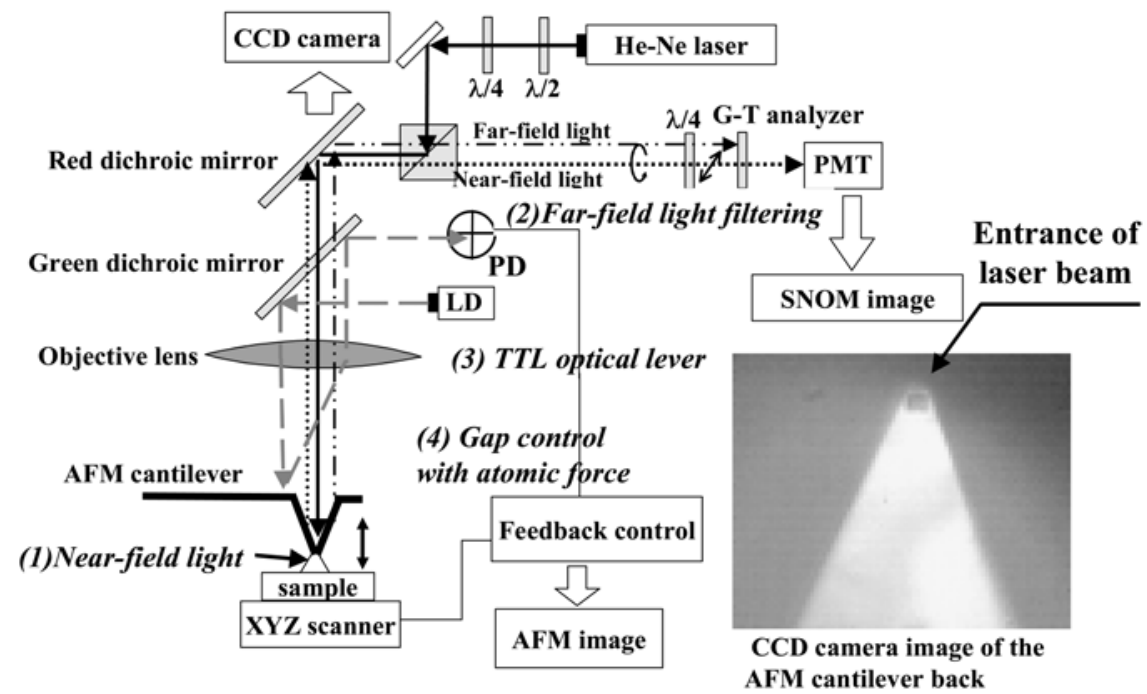

Fig. 6. Scheme of the illumination-collection SNOM system (Hosaka et al., 2007)

resolution was caused by the aperture formed on the top of the metal probe. In Raman spectroscopy, M. Yoshikawa et al. have reported that they developed tuning fork AFM cantilevered illumination-collection type SNOM for stress distribution in VLSI standard sample, which has been used as AFM check sample. The experimental result showed a resolution of about $250 \mathrm{~nm}$ from peak-frequency shift image of the sample around $520 \mathrm{~cm}^{-1}$ Si peak (Fig. 8). These data could not show high spatial resolution of less $50 \mathrm{~nm}$. This might be caused by near-field optical probe with an aperture. Furthermore, the probe has a problem to make a contamination on Si device. Therefore, we have to solve the optical probe with no metal surface probe. We have proposed a plasmon effect near-field optical probe with $\mathrm{Au}$ inner film on outer pyramidal AFM conventional probe made of SiN with no aperture as described in next section. 

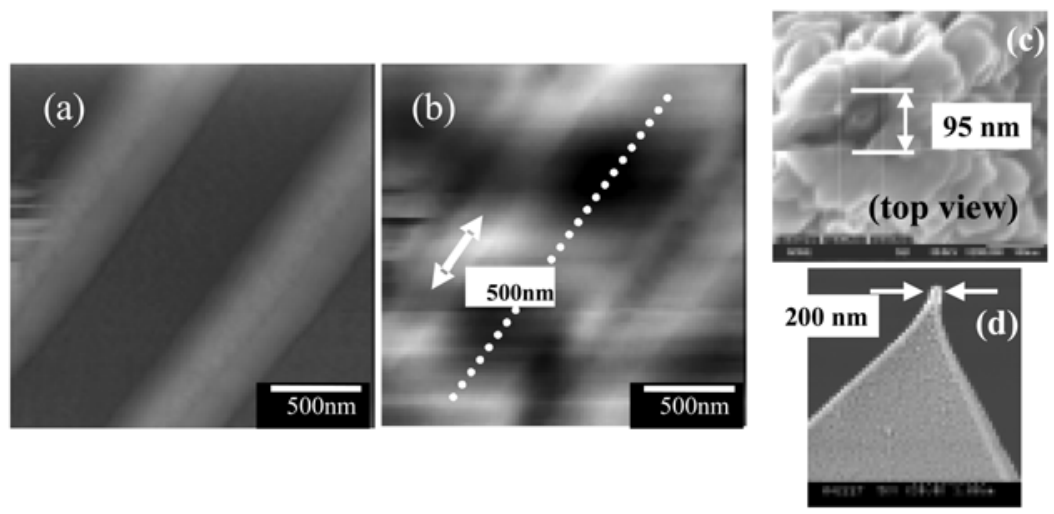

Fig. 7. SNOM observation of 640MB magneto-optical disc(2mmx2mm); (a) AFM imageand (b) SNOM image (Kerr effect image), (c) top view of the aperture and (d)pyramidal probe (Ono et al., 2005).

(a)

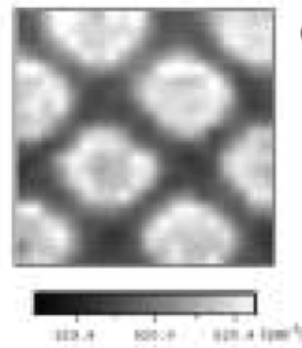

(c)

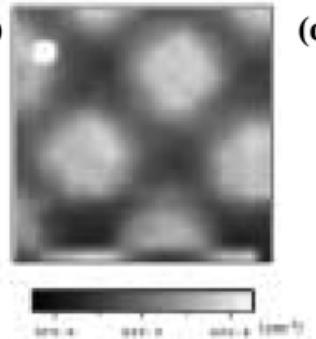

(b)

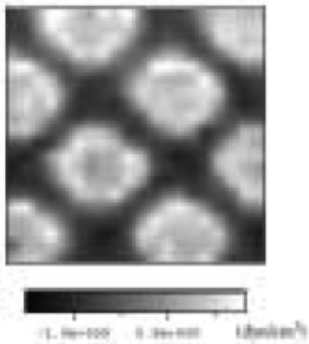

(d)

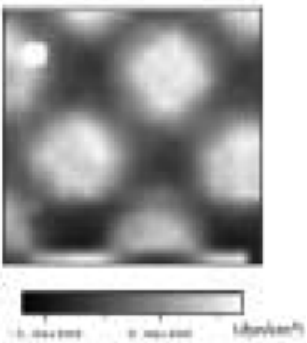

Fig. 8. The near-field Raman scattering Si images of (a) peak-frequency, and (b) stress inVLSI standards, measured by the pyramidical probe with a diameter of $100 \mathrm{~nm}$. Theoptical microscope images of (c) peak-frequency, and (d) stress in VLSI standards,respectively (Yoshikawa et al., 2006),

\section{Illumination-collection mode SNOM with aperture-less pyramidal probe}

In order to consider whether we can obtain fine near-field optical probe from top of the aperture-less pyramidal AFM probe inside-coated with a metal film, we have studied light propagation through the top of probe when illuminating ultra-violet (UV) laser with a wave length of $363.8 \mathrm{~nm}$ using finite differential time domain (FDTD) method. 


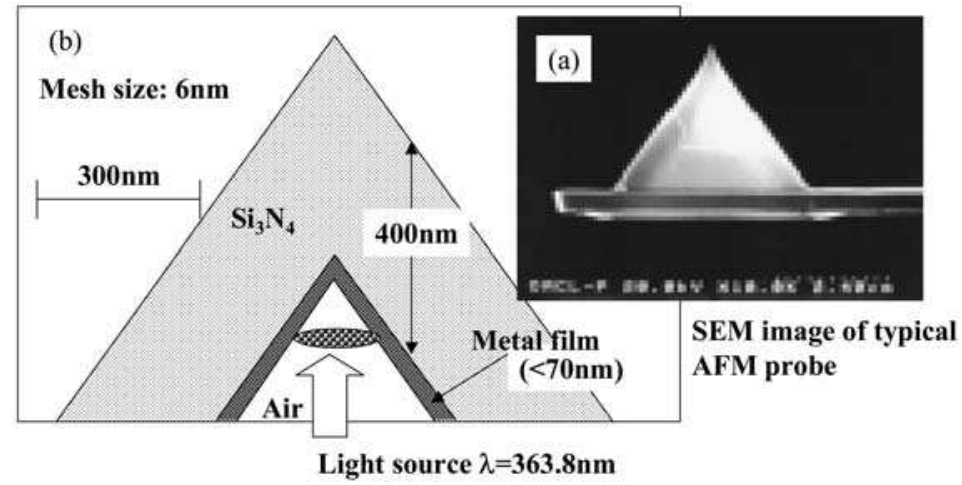

Fig. 9. Calculation model for near-field light emission from aperture-less cantileveredprobe with metal film using FDTD method.

Figure 9 shows the AFM cantilever image and scheme of the probe for SNOM probe. The cantilever is available for commercial pyramidal one, which is model of OMCL-TR400PSA-1 made by Olympus Inc. Figure 9(b) shows the calculation model of an enlarged image of the top of the pyramidal probe based on Fig. 9(a) when illuminating the UV laser into the probe. We executed FDTD calculation with very fine mesh with a size of $6 \mathrm{~nm}$. As a result, we obtained near-field light profiles emitted from the top of the pyramidal probe for various metal films as shown in Fig. 10. The near-field light power becomes strong in order of aluminum, gold and silver. In the cases of $\mathrm{Au}$ and $\mathrm{Ag}$, surface plasmon may occur on the inside metal film. In addition, the near-field light can be propagated along the pyramidal surfaces and emitted from the top. Figure 11 shows the light propagation from the top. The light can propagate to shallow region of $<200 \mathrm{~nm}$ in FWHM of size far from the top.

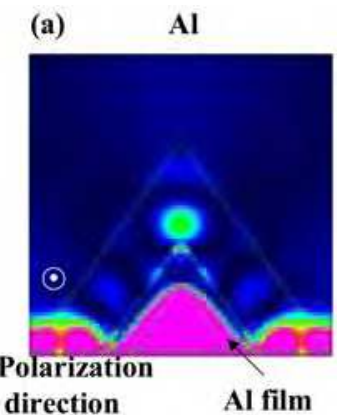

(b)

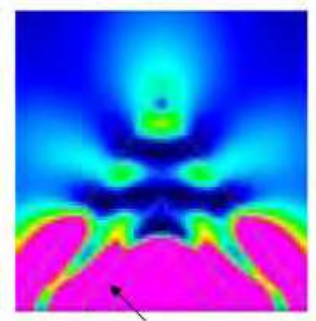

Au film (c) $\quad \mathrm{Ag}$

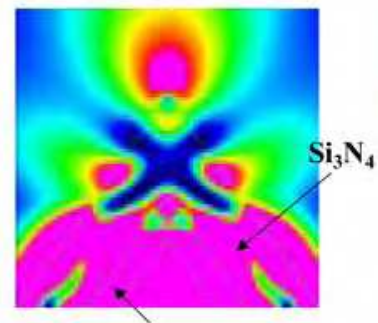

Ag film

Fig. 10. Calculated images of near-field light emission from the top of aperture-less cantilevered probe with various metal films $(50 \mathrm{~nm})$ when illuminating an UV light witha wavelength of $363.8 \mathrm{~nm}$ (Hosaka et al., 2007).

Figure 12 shows calculated results of the near-field light propagation through the top for various Au film thicknesses when illuminating the UV laser. The figure shows that nearfield light emits from the tip through the metal film and SiN dielectric material even by using thick metal film. The power decreases gradually with thickness. The profiles of the optical probe are almost same shape. As described above, we can use the near-field optical 
probe even when using the aperture-less pyramidal probe. The optical profiles, however, indicates that the estimated near-field optical probe size is too large to detect a detail of the light image or localized Raman signal with a resolution of $<100 \mathrm{~nm}$.
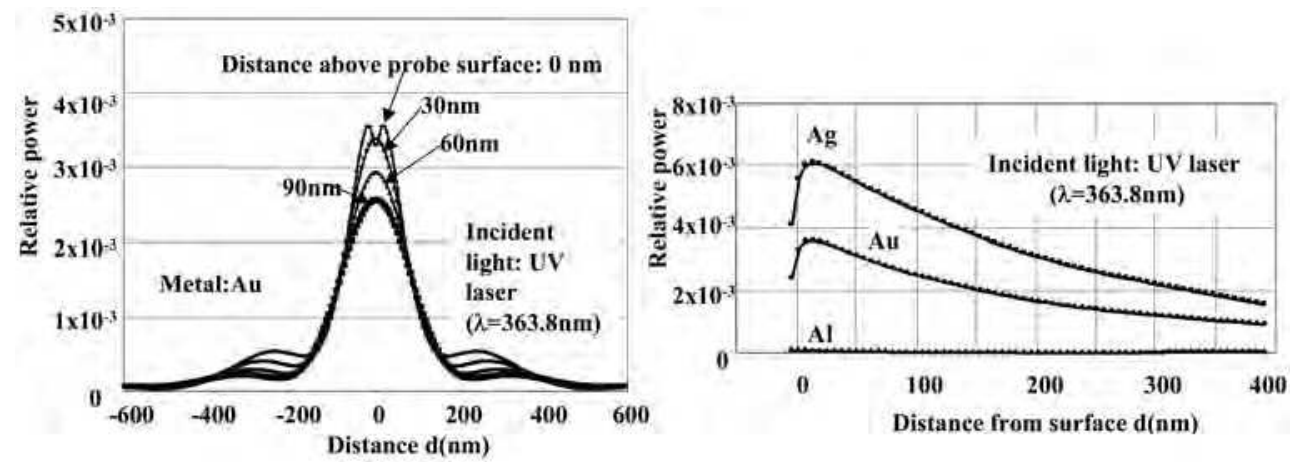

Fig. 11. Calculated results of near-field light propagation for the distances from thesurface of the aperture-less cantilevered probe with various metal film (thickness:50nm), (a) in-plain distribution $n$ and (b).near-field light propagation above the sample surface (Hosaka et al., 2007).
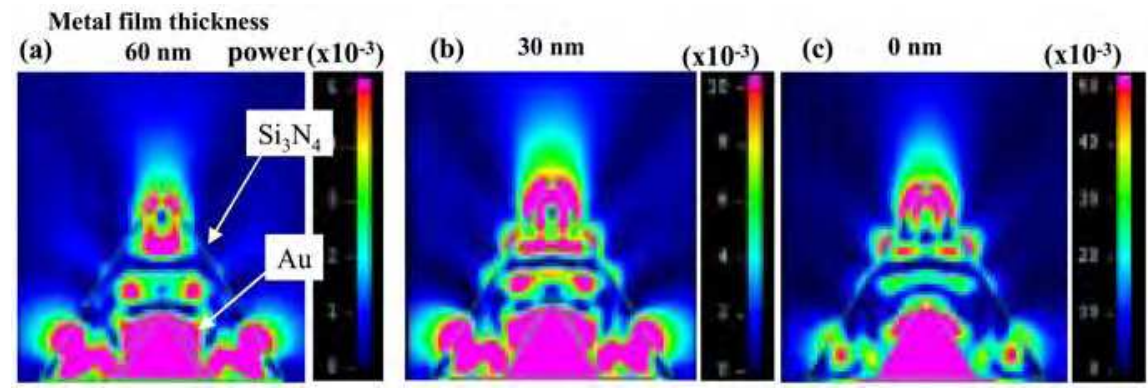

Fig. 12. Calculated images of near-field light emission from the top of aperture-lessmetal probe for various metal film thicknesses, (a) 60 nm-thick Au film, (b) 30 nm-thickAu film, and (c) without the metal film at incident light of UV light with $363.8 \mathrm{~nm}$ in

\section{Prototype atomic force cantilevered SNOM system (Ono et al., 2005)}

The prototyped SNOM system has 4 functions; (1) to keep the gap between the probe and the sample constant by controlling a Z-position of the sample using atomic force detected with the through the lens (TTL) type optical lever6) (AFM function), (2) to generate near-field light emitted from the top of the probe by illuminating the laser beam into the inside of the pyramidal probe (illumination-collection mode SNOM function), (3) to detect only nearfield light reflected from the sample surface using a polarization (depolarization mode SNOM function) or Raman spectroscopy, and (4) to adjust the laser beams incident on the fixed positions of the cantilever using a charge-coupled device (CCD) camera for optical lever and near-field light adjustments. In order to achieve these functions, we have developed multi-beam optics. These optical axes coincide on the object lens. The main optics 
is shown in Fig. 6. In SNOM or Kerr effect detection, a He-Ne laser beam with a wavelength of $632.8 \mathrm{~nm}$ (red laser) and a semiconductor laser beam with $532 \mathrm{~nm}$ (green laser) for nearfield light and atomic force detection of deflection optical lever, respectively, were used. In Raman spectroscopy, UV laser with a wavelength of $363.8 \mathrm{~nm}$ was used with Ar ion laser. The objective lens was used with a numerical aperture (NA) of around 0.5 in both cases. The depolarization optics consists of a He-Ne laser source, a half wave $(\lambda / 2)$ plate, quarter wave $(\lambda / 4)$ plates, AFM cantilever with a small aperture, a G-T analyzer, and a photomultiplier tube (PMT). The linearly polarized light that emitted from the He-Ne laser is converted into circularly polarized light through the $\lambda / 4$ plate, and is focused into the inside of the probe on the cantilever tip. After the polarized near-field light is reflected from the sample surface through or outside of the top, a plane of polarization is slightly rotated. By passing the reflected light through the $\lambda / 4$ plate, the plane of the polarization is converted to the linearly polarized light with various angles. The G-T analyzer is adjusted to remove the far-field light reflected from inside wall of the probe. On the other hand, nearfield light reflected from the sample surface has a little shift of polarization so that we can detect only polarization-rotated near-field light using G-T analyzer.

Figure 13 shows AFM and depolarization SNOM images of the sputtered Au film on the glass with a comparison of SEM image. The system observed not only many fine clacks in the film in AFM image, but also bright small lines with a width of $<10 \mathrm{~nm}$ in SNOM image. The images indicate that both functions of AFM and SNOM have very fine resolution of less than $10 \mathrm{~nm}$.
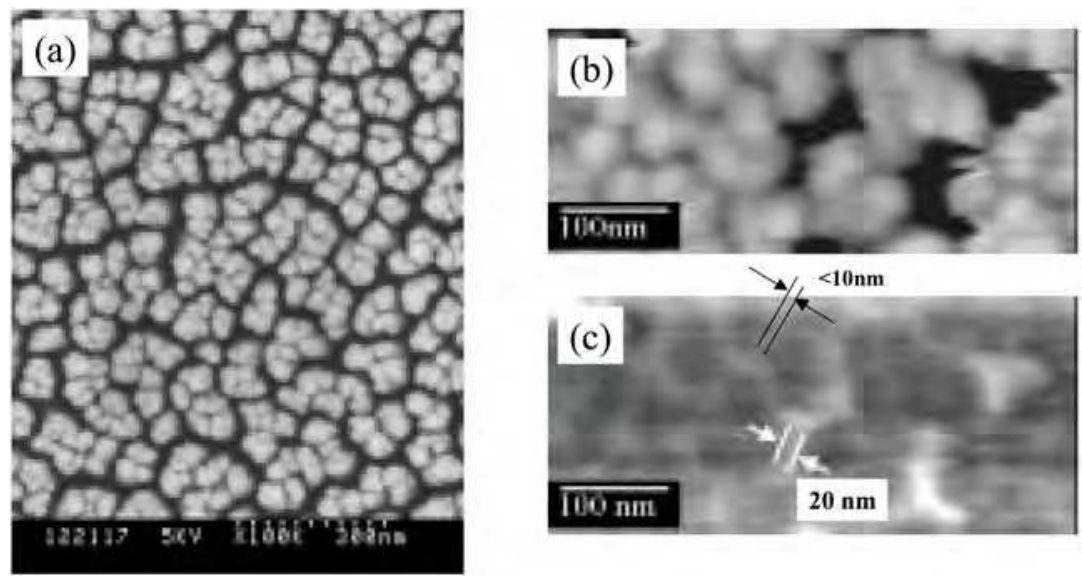

Fig. 13. Test sample observation of Au film; (a) SEM image, (b) AFM image and (c) SNOM image (Hosaka et al., 2007).

The optical system observed Kerr effect near field image of optical recording of conventional giga magneto-optical (MO) disc as shown in Fig. 14. From the rise-up at the signal edge, a resolution of Kerr effect image was less than $20 \mathrm{~nm}$, considering a magnetic domain wall between switched bits. Comparing these data with previous image (Fig. 7), the aperture-less pyramidal SNOM has the potential to achieve fine spatial resolution in near-field light images. 


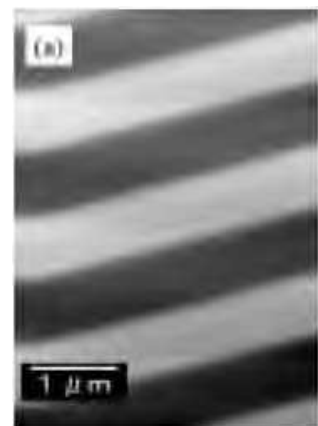

AFM像

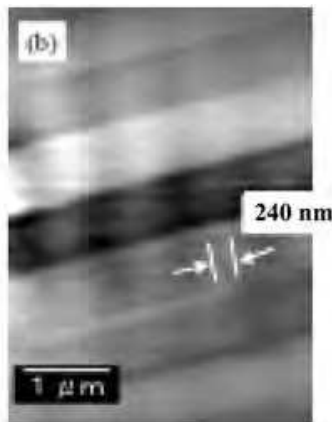

Polarized SNOM像

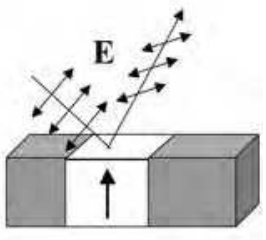

Kerr effect

(Kerr rotation)

Fig. 14. AFM and SNOM images of 2.3 GB-MO recorded bits (MO disc spec.: min. bit length 233nm, track pitch: $670 \mathrm{~nm}$ (Hosaka et al., 2007).

\section{Combination of SNOM and Raman spectroscopy (Hosaka et al. 2007)}

We have prototyped the combination system with above system (Fig. 6) and Raman spectroscopy of Nanofinder30, which is made by Tokyo Instruments Inc., as shown in Fig. 15. The stimulated laser was used with UV line with a wavelength of $363.8 \mathrm{~nm}$ in Ar ion laser. Figure shows a scheme of optical functions of the system. In practice, the spectroscopy was inserted between the TTL type optical lever system and the depolarization optics. The sample with gates and shallow trench insulator (STI) on Si substrate was prepared for measuring its stress distribution.

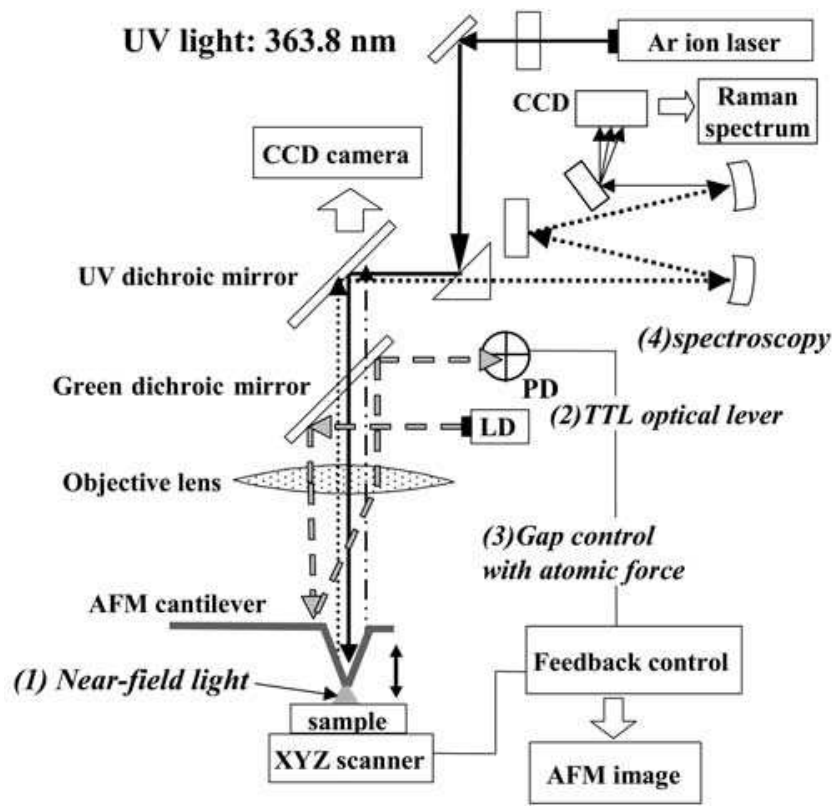

Fig. 15. Scheme of prototyped SNOM Raman spectroscopic microscopy (Hosaka et al., 2007). 
Figure 16 shows Raman spectra change before and after AFM operation when illuminating the UV laser into the inside of aperture-less pyramidal probe. Figure 16(a) shows the spectrum under the sample was far from the probe. The Si Raman peak disappeared. Under controlling the system in the contact mode AFM, the peak at about $520 \mathrm{~cm}^{-1}$ was obtained. When adjusting the optics and using strong power of UV laser, strong signal was obtained as shown in Fig. 16(c).
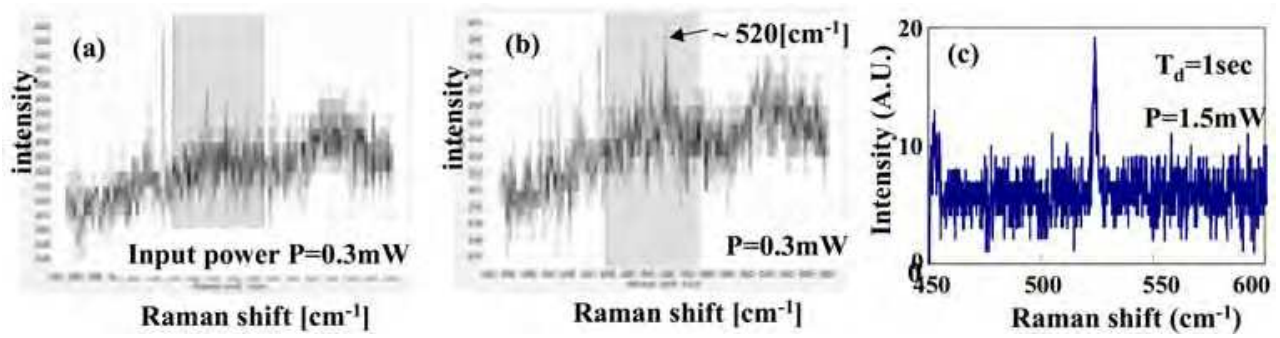

Fig. 16. Raman shift spectra of the silicon sample when illuminating UV light with a wavelength of $363.8 \mathrm{~nm}$ to inside of the pyramidal probe, (a) in separation between the probe and the sample, (b) under contact mode controlling at $20 \mathrm{nN}$, and (c) incident laser power of $1.5 \mathrm{~mW}$ (Hosaka et al., 2007).

Figures 17(a) and 17(b) show spectra detected by the near-field optical Raman spectroscopy (NFRS) and optical microscopic Raman spectroscopy (OMRS) at an accumulation time of 16 sec, respectively. The OMRS can be easily achieved by removing the cantilever probe. Although the detected intensity of NFRS is weaker than that of OMRS, it is clarified that we detected Raman scattering peak of Si using the system and the aperture-less pyramidal probe.

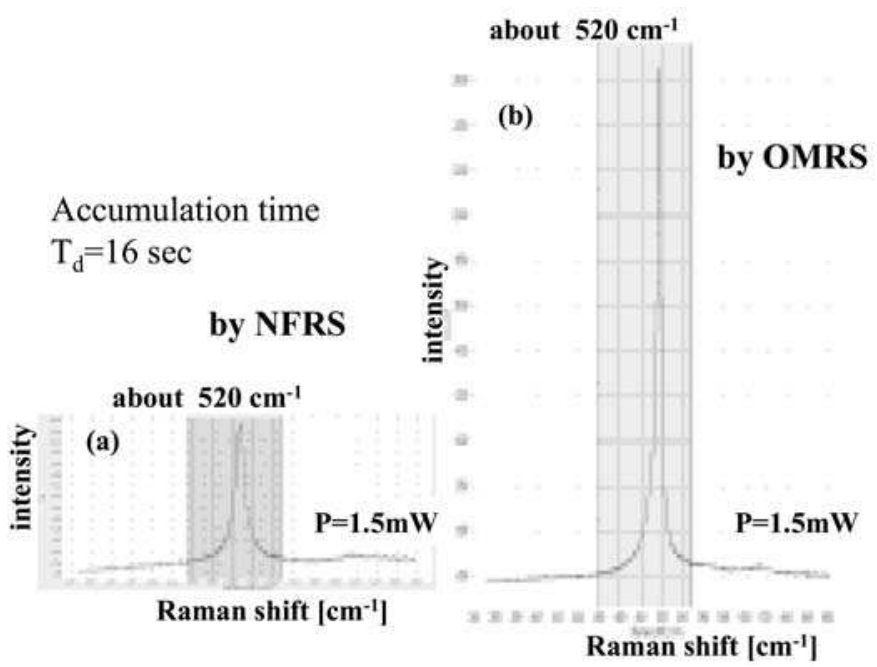

Fig. 17. Comparison of spectra in near-field optical Raman spectroscopy (NFRS) (a) andoptical microscopy Raman spectroscopy (OMRS) (Hosaka et al., 2007). 


\section{Raman spectroscopy of Si device test sample (Hosaka et al.)}

The test sample structure and anticipated Raman peak shift of Si are shown in Fig. 18. The compression and tensile stresses distribution can be estimated in the sample. In the gate, compression stress occurs because of oxidation of both gate sides. In the Si area between the STI and the gate, tensile stress ocurrs because $\mathrm{STI}_{-} \mathrm{SiO}_{2}$ volume is shrunk by annealing.

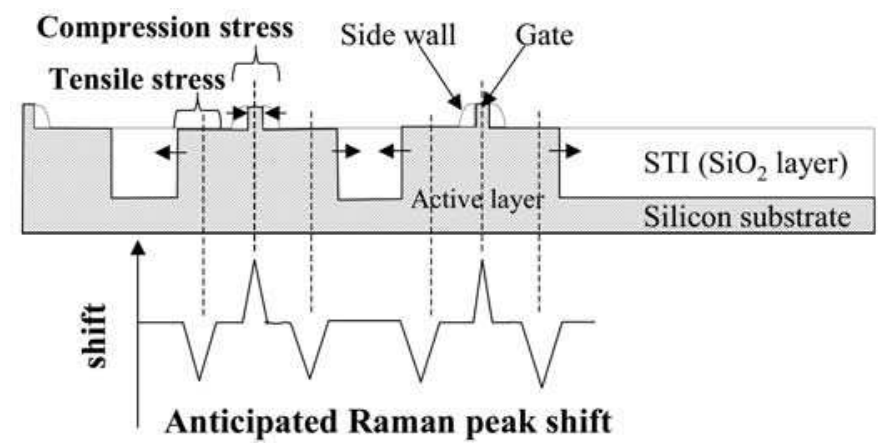

Fig. 18. A structure of the test sample, and anticipated stress model and Raman peak shift.

Using the sample, the surface structure was observed by AFM function in prototyped SNOM and scanning electron microscope (SEM). We can observe small dimension of the gate of about $25 \mathrm{~nm}$ as shown in Fig. 19. Then, we measured Raman spectrum around 520 $\mathrm{cm}^{-1}$ at each pixel on one line of the sample for peak-frequency shift and stress distribution.
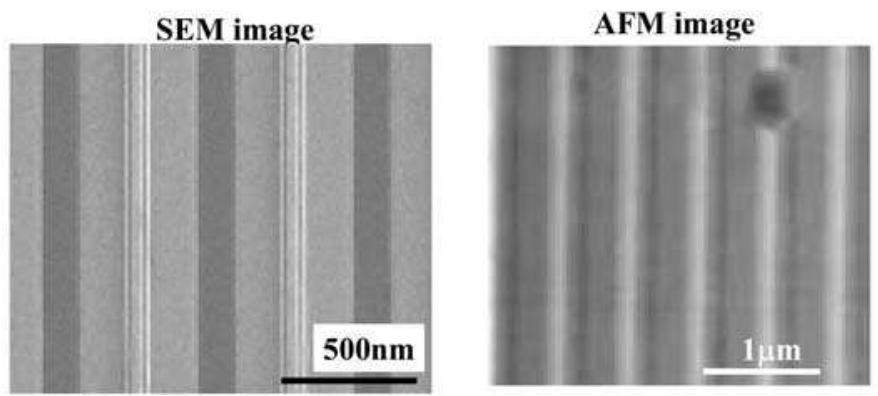

Fig. 19. SEM and AFM images of the sample, AFM image was taken by SNOM system. and stress model.

After the measurement, the peak-frequency shift was processed by Lorenz peak fitting of the spectrum for fine spatial resolution of less than $0.1 \mathrm{~cm}^{-1}$. The peak-frequency shift profile is shown in Fig. 20. The profile is well agreed with the anticipated profile. The peak of compress stress appears at the gate part. The FWHM of the peak is less than $25 \mathrm{~nm}$. The result was obtained when the focused laser beam with a power of $1.5 \mathrm{~mW}$ was illuminated into the pyramidal probe of AFM. The Au film was coated inside the probe with a thickness of $50 \mathrm{~nm}$. The highest compression stress of $1-1.5 \times 10^{4}\left[\mathrm{Ncm}^{-2}\right]$ was estimated, and is consistent with the result reported by M. Yoshikawa st al. Therefore, the technology has the potential to get near-field Raman scattering profile with fine spatial resolution of less than $25 \mathrm{~nm}$. 


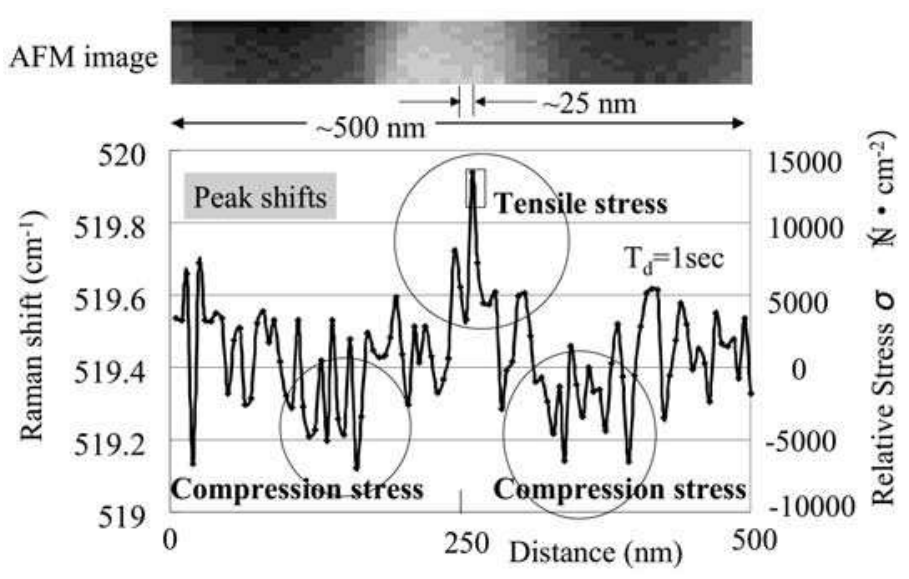

Fig. 20. AFM image of the gate and Raman spectrum peak shift around the gate.

\section{Summary}

Recent technologies of tip-enhanced Raman spectroscopy (TERS) and scanning near-field optical microscopy (SNOM) with Raman spectroscopy are reviewed.

In TERS technology, it has been developed based on surface-enhanced Raman spectroscopy (SERS). The some TERS are reviewed as follows:

1. Bottom illumination mode TERS has been described, and it has demonstrated fine spatial resolution and gigantic enhancement of Raman scattering signal using single wall carbon nano tube (SWNT).

2. Side- and modified top-illumination mode TERS have been described, and they have demonstrated enhancement of Raman scattering signal and resolution of subwavelength.

3. There are, however, some technical issues such as metal contamination, stress measurement of Si device, etc. This means that it is difficult to apply to an evaluation of semiconductor devices, etc.

In SNOM technologies, illumination-collection mode SNOM has been described with regard of aperture type SNOM probe and aperture-less pyramidal SNOM probe.

4. Using the aperture type SNOM probe, it is difficult to obtain fine spatial resolution because the aperture makes only near-field optical probe incompletely.

5. M. Yoshikawa et al. demonstrated spatial resolution of about $200 \mathrm{~nm}$ in peak-frequency shift image with Raman Si peak of VLSI standard sample using illumination-collection mode with the aperture type SNOM probe.

6. S. Hosaka et al. have proposed the aperture-less pyramidal probe in illuminationcollection mode SNOM to improve near-field optical probe and to protect from the metal contamination to the device surface. The SNOM and Raman spectroscopy was combined with UV laser. The prototyped microscopy demonstrated following possibilities.

a. By calculating near-field light propagations in the aperture-less pyramidal probe by FDTD method, strong near-field light propagation from top of the aperture-less 
pyramidal probe occurs due to surface plasmon effect using $\mathrm{Au}$ and $\mathrm{Ag}$ as inside metal film.

b. Very fine SNOM image of the crack network of less than $10 \mathrm{~nm}$ in thin Au film using the aperture-less pyramidal probe SNOM was obtained with a spatial resolution of less than $10 \mathrm{~nm}$.

c. The combination system of SNOM and Raman spectroscopy has the possibility to detect Raman scattering light using the aperture-less pyramidal probe.

d. The system measured the Si sample with STI and gate structure to get both AFM image and Si peak-frequency shift in stokes scattering.

e. The system observed compression and tensile stress of $1-1.5 \times 10^{4}\left[\mathrm{Ncm}^{-2}\right]$ on the sample.

f. The spatial resolution of less than $25 \mathrm{~nm}$ was demonstrated in Raman spectroscopy. As described above, scanning near-field Raman spectroscopic microscopy has the potential to measure a detail of the sample such as structure, magnetization, optical property, chemical characterization, stress distribution, etc with a fine spatial resolution of $25 \mathrm{~nm}$. In the future, the technology is expected to be one of key technologies for evaluation of materials.

\section{References}

Durig, U., Pohl, D. W. and Rohner, F. (1986). J. Appl. Phys. Vol. 59, 3318.

Fischer, U. C. and Pohl D. W. (1989). Phy. Rev. Lett. Vol. 62, 458.

Hartschuh, A., Anderson, N. and Novotny, L. (2003). J. Microscopy, Vol. 210, 234-240.

Hayazawa N, Inouye Y, Sekkat Z, Kawata S. (2002). J. Chem. Phys. Vol. 117, 1296.

Hayazawa N, Inouye Y, Tarun A, Kawata S. (2002). J. Appl. Phys. Vol.92, 6983.

Hosaka, S, Shintani, T, Miyamoto, M, Kikukawa, A, Hirotsune, A, Terao, M, Yoshida, M, Fujita, K, Kammer, S. (1996). J. Appl. Phys., Vol. 79, 8082.

Hosaka, S., Shintani, T., Kikukawa, A. and Itoh, K. (1999). J. Microscopy, Vol.194 , 369-373.

Hosaka, S., Sone, H., Takahashi, Y., Shintani, T., Kato, K., Saiki, T. (2003). Microelectronic Engineering, Vol. 67-68, 728.

Hosaka, S., Shimizu, T., Mine, K., Shimada, K., and Sone, H. (2007) . J. Phys.: Conf. Ser. Vol. 61, 425-429.

Hosaka, S., Koyabu, H., Mine, K., Aramomi, Y., Sone, H., Sato, E., Tochigi, K. (2007). Proc. 2007 IEEE Int. Conf. Electron Devices and Solid-State Circuits Tainan, Taiwan, pp. 297300.

Hosaka, S., Koyabu, H., Mine, K., Aramomi, Y., Sone, H., Sato, E., Tochigi, K. to be submitted.

Nieman L.T., Krampert G.M., Martinez R.E. (2001). Rev. Sci. Instrum. Vol. 72, 1691.

Ono, M., Sone, H., and Hosaka, S. (2005). Jpn. J. Appl. Phys. Vol. 44, 5434.

Poborchii V., Tada T., Kanayama T. (2005). Jpn. J. Appl. Phys. Vol. 44, L202.

Pohl, D. W. (1986). IBM J. Res. Develop. Vol. 30, 417.

Rim, K., Hoyt J.L., Gibbons J.F. (2000). IEEE Transactions on Electron Devices, Vol. 45, 14061415.

Stockle R., Suh Y.D., Deckert V., Zenobi R. (2000) Chem. Phys. Lett. Vol. 318, 131.

Synge, E.H. (1932). Philos. Mag. Vol. 13: 297.

Tezuka, T., Sugiyama, N., and Takagi, S. (2001). Appl. Phys. Lett. Vol. 79, 1798-1800 . 
Trautman J.K.,Macklin J.J., Brus L.E, Betzig E. (1994). Nature, Vol. 369, 40.

Welser, J., Hoyt, J.L., Gibbons, J.F. (1994). Electron Device Letters, Vol. 15, 100-102.

Wessel J. (1985).J. Opt. Soc. Am. B, Vol. 2, 1538.

Yoshikawa, M, Murakami, M, Matsuda, K, Sugie, R. Ishida, H, Shimizu, R, Jpn. J. Appl. Phys. 45, L486 (2006). 


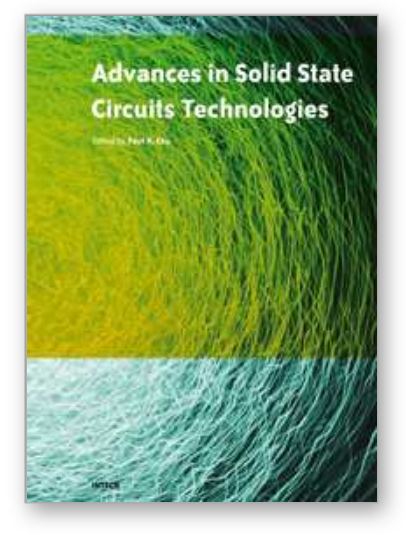

\author{
Advances in Solid State Circuit Technologies \\ Edited by Paul K Chu
}

ISBN 978-953-307-086-5

Hard cover, 446 pages

Publisher InTech

Published online 01, April, 2010

Published in print edition April, 2010

This book brings together contributions from experts in the fields to describe the current status of important topics in solid-state circuit technologies. It consists of 20 chapters which are grouped under the following categories: general information, circuits and devices, materials, and characterization techniques. These chapters have been written by renowned experts in the respective fields making this book valuable to the integrated circuits and materials science communities. It is intended for a diverse readership including electrical engineers and material scientists in the industry and academic institutions. Readers will be able to familiarize themselves with the latest technologies in the various fields.

\title{
How to reference
}

In order to correctly reference this scholarly work, feel free to copy and paste the following:

Sumio Hosaka (2010). Scanning Near-Field Raman Spectroscopic Microscope, Advances in Solid State Circuit Technologies, Paul K Chu (Ed.), ISBN: 978-953-307-086-5, InTech, Available from:

http://www.intechopen.com/books/advances-in-solid-state-circuit-technologies/scanning-near-field-ramanspectroscopic-microscope

\section{INTECH}

open science | open minds

\section{InTech Europe}

University Campus STeP Ri Slavka Krautzeka 83/A 51000 Rijeka, Croatia

Phone: +385 (51) 770447

Fax: +385 (51) 686166 www.intechopen.com

\section{InTech China}

Unit 405, Office Block, Hotel Equatorial Shanghai No.65, Yan An Road (West), Shanghai, 200040, China 中国上海市延安西路65号上海国际贵都大饭店办公楼405单元 Phone: +86-21-62489820

Fax: $+86-21-62489821$ 
(C) 2010 The Author(s). Licensee IntechOpen. This chapter is distributed under the terms of the Creative Commons Attribution-NonCommercialShareAlike-3.0 License, which permits use, distribution and reproduction for non-commercial purposes, provided the original is properly cited and derivative works building on this content are distributed under the same license. 